\title{
$-1$ \\ Intraplaque hemorrhage in symptomatic and asymptomatic progressive carotid artery stenosis - pilot study
}

\author{
'Školoudík D, ${ }^{1}$ Roubec M, ${ }^{2}$ Herzig R, ${ }^{3}$ Havelka J
}

1 Ostrava University and University Hospital, Department of Neurology, Ostrava, Czech Republic

2 Charles University Faculty of Medicine and University Hospital Hradec Králové, Department of Neurology, Hradec Králové, Czech Republic

3 Ostrava University and University Hospital, Department of Radiology, Ostrava, Czech Republic

Background and aims: Intraplaque hemorrhage (IPH) belongs to potential mechanisms of unstable plaque development. Study aims to compare the IPH occurrence in patients with asymptomatic stable (ASS), asymptomatic progressive (APS) and symptomatic (SS) carotid artery stenosis $>50 \%$.

Materials and methods: Serial duplex ultrasound (DUS) in a 6-month period and magnetic resonance imaging (MRI) using axial 3DT1w sequence were used for IPH detection in patients with carotid stenosis. Stenoses in patients with ipsilateral stroke/transient ischemic attack within previous 4 weeks or acute ischemic lesion on diffusion-weighted MRI were evaluated as symptomatic. Stenoses with progression of $>10 \%$ since last DUS examination were evaluated as progressive. Echolucent part of atherosclerotic plaque $>8 \mathrm{~mm}^{2}$ on DUS (Fig.1) and hyperintensity on 3DT1w-MRI (Fig.2) were evaluated as IPH. Differences in IPH occurrence between ASS, APS and SS patients were statistically evaluated.

Results: Totally 32 patients (18 males, mean age $71.3 \pm 7.7$ years) were enrolled during 18 months; 5 patients with ASS, 18 with APS and 9 with SS. MRI examination was not performed in 1 SS patient. IPH was detected using DUS/MRI in $1(20 \%) / 2(40 \%)$ of ASS patients, $9(50 \%) / 8(53 \%)$ of APS patients, and $5(56 \%) / 4(50 \%)$ of SS patients ( $p>0.05$ in all cases). IPH on both DUS and MRI were detected in none of ASS patients, 5 (28\%) APS patients and $3(33 \%)$ SS patients ( $p>0.05$ in all cases).

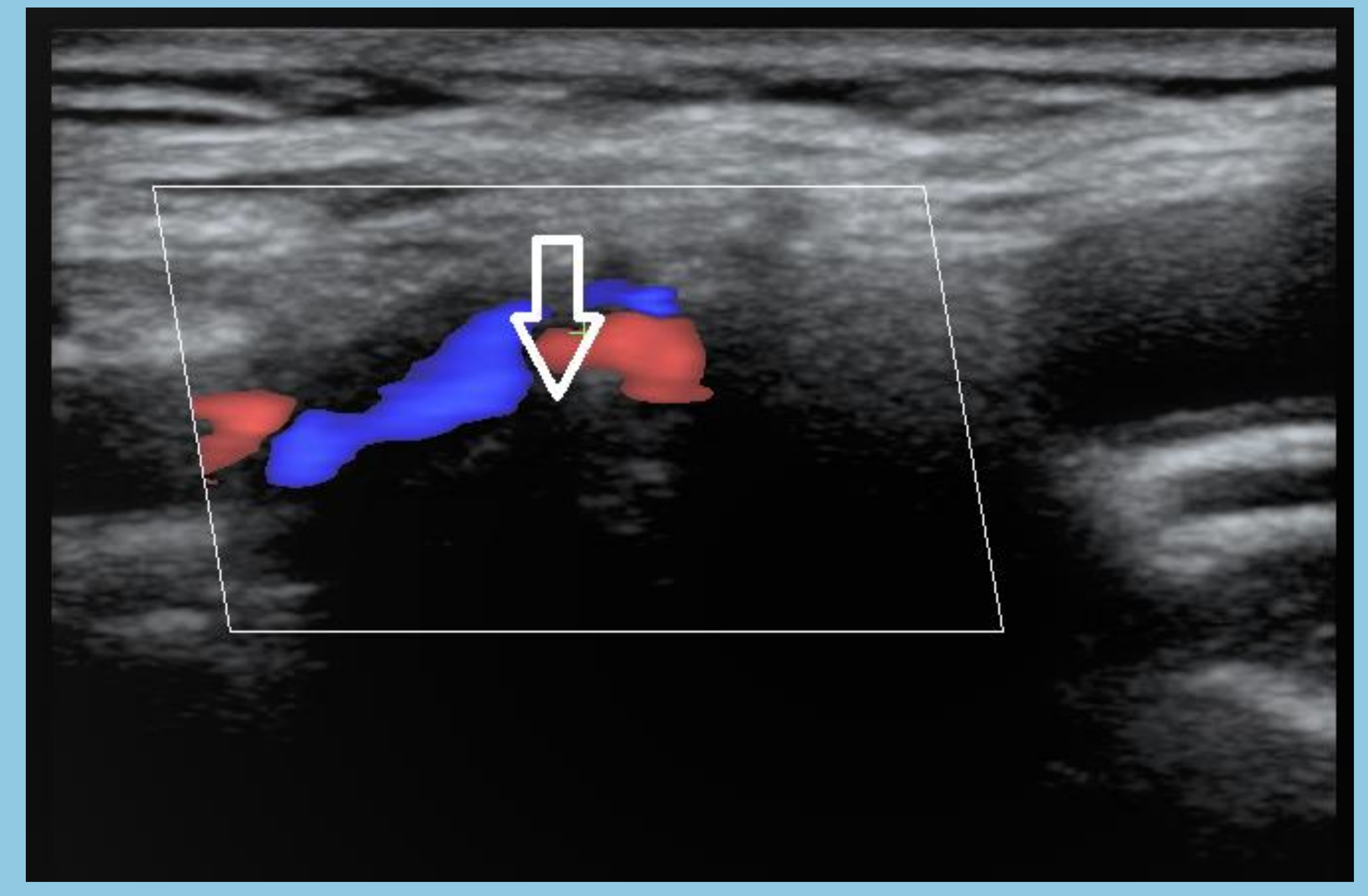

Fig. 1. IPH on DUS

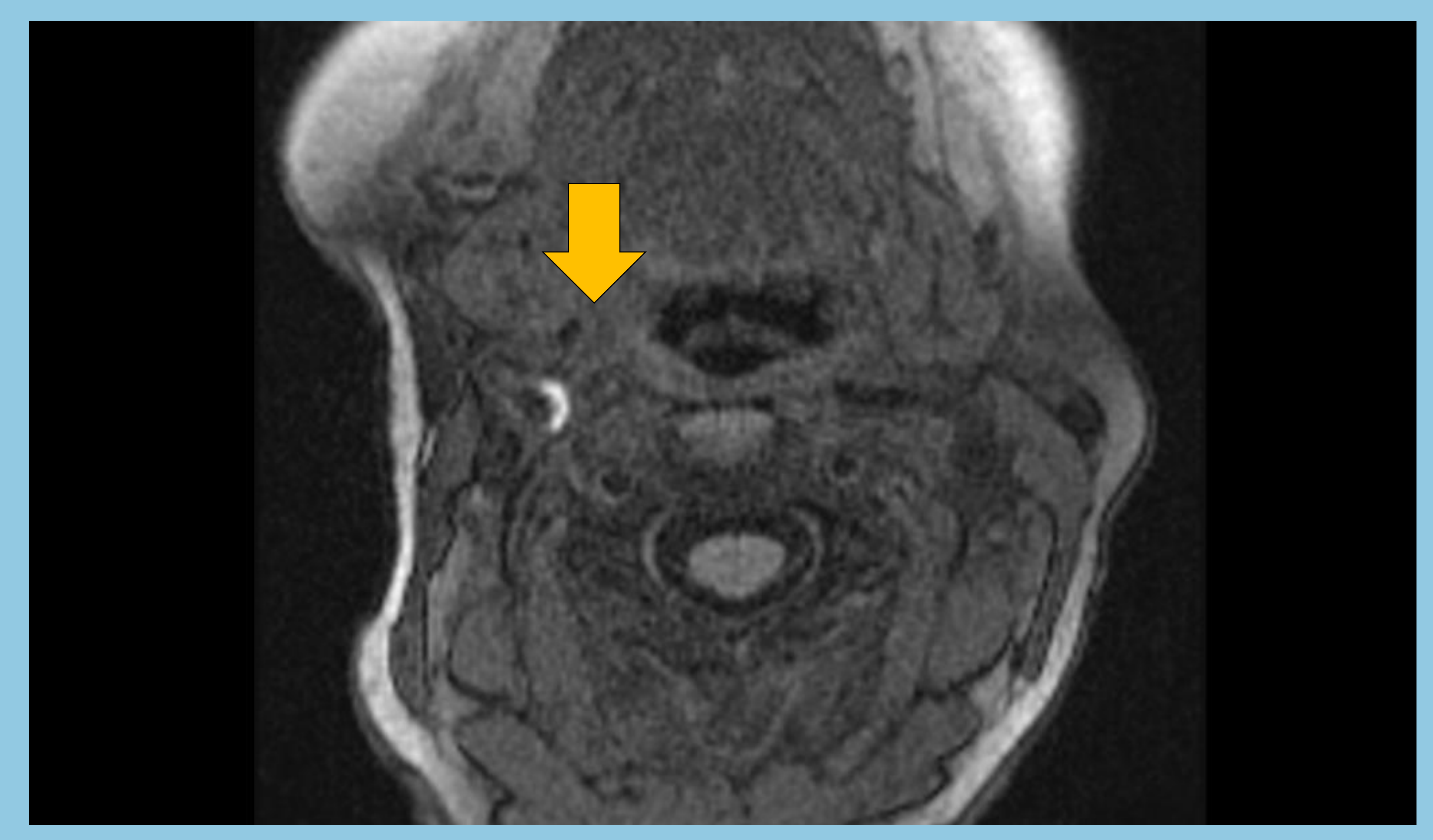

Fig. 2. IPH on MRI

Conclusion: No significant difference in IPH occurrence was found between ASS, APS and SS patients in the pilot study group - small number of subjects. Combination of DUS and MRI shows positive trend in detection of IPH with risk of progression or stroke (APS and SS). Totally 200 patients will be enrolled to the ongoing study. 